\title{
Comment on "PIC simulations of circularly polarised Alfvén wave phase mixing: a new mechanism for electron acceleration in collisionless plasmas" by Tsiklauri et al. (Research Note)
}

\author{
F. Mottez ${ }^{1, \star}$, V. Génot ${ }^{2}$, and P. Louarn ${ }^{2}$ \\ ${ }^{1}$ Centre d'étude des Environnements Terrestre et Planétaires, 10-12 Av. de l'Europe, 78140 Vélizy, France \\ e-mail: fabrice.mottez@cetp.ipsl.fr \\ 2 Centre d'Etude Spatiale des Rayonnements, 9 Av. Colonel Roche, 31400 Toulouse, France
}

Received 20 September 2005 / Accepted 18 November 2005

ABSTRACT

Tsiklauri et al. recently published a theoretical model of electron acceleration by Alfvén waves in a nonuniform collisionless plasmas. We compare their work with a series of results published earlier by an another team, of which Tsiklauri et al. were probably unaware. We show that these two series of works, apparently conducted independently, lead to the same conclusions. This reinforces the theoretical consistency of the model.

Key words. Sun: oscillations - Sun: Corona - Sun: solar wind - plasmas - acceleration of particles

Two recent papers published by Tsiklauri et al. $(2005 \mathrm{~b}, \mathrm{a})$, based on kinetic numerical simulations, show a mechanism of astrophysical relevance that could explain the acceleration of electrons in a magnetized collisionless plasma.

The idea is the following: Alfvén waves (AW) are often observed or inferred in astrophysical plasmas; it has been theoretically demonstrated that they could be the source of plasma acceleration. In parallel propagation (i.e. with a wave vector aligned with the local magnetic field) an AW carries no parallel electric field, and therefore cannot accelerate particles in that direction. However when the wave is structured in the perpendicular direction, i.e. when $k_{\perp} \neq 0$ and sufficiently large, the AW acquires a significant parallel electric field component. Oblique AW can form when an initially parallel AW propagates in regions with perpendicular Alfven velocity (or density) gradients. Such a situation leads to a distortion of the wavefronts: small perpendicular length scales are formed and finally a parallel electric field component appears.

Although not new as claimed by the authors, this mechanism is, in our opinion, interesting. In this comment, the results exposed by Tsiklauri et al. (2005a) are briefly discussed and compared with other works on the same subject published earlier by Génot et al. (1999); Génot et al. (2000, 2001, 2004).

* Now at LUTH, Observatoire de Paris, 5 place Jules Janssen, 92195 Meudon, France.
Génot et al. investigated the acceleration of electrons in the Earth's aurora. Following in-situ exploration by satellites, the well-constrained observations make this region appropriate for the testing of theoretical models. Génot et al. chose a plasma with a high magnetic field, given by a ratio of the electron cyclotron to the electron plasma period $\omega_{\mathrm{ce}} / \omega_{\mathrm{pe}}=4$, as in the Earth auroral zone. Because plasma cavities are observed in connexion with electron acceleration and plasma turbulence, the gradients delimit a density depletion. Tsiklauri et al. (2005a) refer to electron acceleration in solar open coronal structures, the magnetic field is weaker $\omega_{\mathrm{ce}} / \omega_{\mathrm{pe}}=1$ and they consider a density bump (expected, for instance, at the boundary of a coronal loop).

Do the two works explore fundamentaly different regimes? Goertz (1984), using the Maxwell-Vlasov equations, performed a linear study of the oblique AW propagation. $\mathrm{He}$ showed that the value of the parallel electric field depends on the dimensionless parameter $r=\beta m_{\mathrm{i}} / m_{\mathrm{e}}$ with respect to one. ( $\beta=2 \mu_{0} p / B^{2}$ is the ratio of the kinetic plasma pressure to the magnetic pressure, and $m_{\mathrm{i}} / m_{\mathrm{e}}$ is the ion to electron mass ratio.) In Génot et al. $m_{\mathrm{i}} / m_{\mathrm{e}}=100, \beta \sim 800^{-1}$ and $r=0.125$. In Tsiklauri et al. $m_{\mathrm{i}} / m_{\mathrm{e}}=16, \beta \sim 50^{-1}$ and $r=0.32$. All the publications discussed in this comment deal with the same regime, $r<1$, the inertial AW regime. 
Génot et al. (1999) made a linear study of the wave propagation in a transverse gradient (for $r<1$ ), and showed that to create a parallel electric field, the characteristic size of the gradient must be of the order of the electron inertial length $c / \omega_{\text {pe }}$. The simulations carried out by Tsiklauri et al. and by Génot et al. are all in this regime; this allows for almost direct comparisons.

Tsiklauri et al. (2005b) compare their results to those of Hasegawa \& Chen $(1975,1976)$. Many conclusions seem to agree, and we expect that the differences (appart from non homogeneity, not treated by Hasegawa \& Chen) are due to the parameter $r$ that is greater than one in Hasegawa \& Chen (1975, 1976). When $r>1$, the characteristic transverse length is not $c / \omega_{\text {pe }}$ but the ion Larmor radius.

The PIC simulation codes used by Génot et al. and Tsiklauri et al. are slightly different. In Tsiklauri et al. (2005b,a), the code describes the full dynamics of the electrons, and requires a small time step (a run requires 8 days). In Génot et al. (2000); Génot et al. (2001); Génot et al. (2001, 2004), the code describes the guiding centre dynamics of the electrons, and if $\omega_{\mathrm{ce}} / \omega_{\mathrm{pe}}>1$, it allows for larger time steps. A simulation typically lasts $10 \mathrm{~h}$.

The initial conditions are very similar in Tsiklauri et al. (2005a) and Génot et al. (1999): a local perturbation is propagated by parallel Alfvén waves. Tsiklauri et al. show that the AW propagation in their kinetic simulation is like in MHD. In the other works conducted by Génot et al. (2000); Génot et al. (2001); Génot et al. (2004), the simulations are initialised with bi-fluid (therefore almost MHD) Alfvén waves filling the whole simulation domain. The kink in the ion velocities, observed by Tsiklauri et al. is set initially by Génot et al. Such an initialization allows one to control the direction of propagation of the wave. Génot et al. (2000) could show that the electron acceleration occurs most efficiently in the direction of propagation of the AW; the bulk velocity is translated in the direction of propagation of the wave. But some electrons are (much less) accelerated in the reverse direction. As the Alfvén velocity is, in our regime, much faster than the electron thermal velocity, we consider that the acceleration process is non-resonnant (and therefore not a Landau process), even if second-order resonance effects give more efficient acceleration in the direction of the wave (Génot et al. 2001).
The parallel electric field (Génot et al. 2000, 2001; Génot et al. 2004) shows a spiky structure superimposed on a smooth and less intense electric field. The smooth electric field is created in the linear phase of the AW dissipation process (Génot et al. 1999), as can be seen in simulations (Génot et al. 2001). As this field exists over large distances along the magnetic field, it has a high potential for accelerating electrons. Then, when the acceleration begins, many strong and localized quasielectrostatic high amplitude spikes develop. These spikes are probably the structures seen on the $E_{x}$ displayed in Tsiklauri et al. (2005a). Their nature and origin have been extensively studied (Génot et al. 2001, 2004). They are the consequence of the nonlinear interaction of the beams of accelerated electrons and current densities with the bulk of the plasma (beam-plasma and Buneman instabilities). These spikes have the same characteristics as those observed near the plasma cavities by auroral satellites. If this process is indeed correctly understood, they are a consequence, and not the cause of the electron acceleration (Génot et al. 2004).

As the magnetic field is lower in the simulations conducted by Tsiklauri et al., the Alfvén wave velocity is smaller, closer to the electron thermal velocity, and it is possible that resonnant effects are more important in the wave particle interaction.

In spite of some minor differences of regime and minor divergences in the interpretation of the simulation results, the rediscovery of the acceleration process initially proposed by Génot et al. and the similarity of the most important results strenghtens the theoretical relevance of this model.

\section{References}

Génot, V., Louarn, P., \& Mottez, F. 2000, J. Geophys. Res., 105, 27611 Génot, V., Louarn, P., \& Mottez, F. 2001, J. Geophys. Res., 106, 29633 Génot, V., Louarn, P., \& Mottez, F. 2004, Ann. Geophys., 22, 2081

Génot, V., Louarn, P., \& Le Quéau, D. 1999, J. Geophys. Res. (Space Phys.), 104, 22649

Génot, V., Mottez, F., \& Louarn, P. 2001, Phys. Chem. Earth C, 26, 219

Goertz, C. K. 1984, Planet. Space Sci., 32, 1387

Hasegawa, A., \& Chen, L. 1975, Phys. Rev. Lett., 35, 370

Hasegawa, A., \& Chen, L. 1976, Phys. Fluids, 19, 1924

Tsiklauri, D., Sakai, J.-I., \& Saito, S. 2005a, A\&A, 435, 1105

Tsiklauri, D., Sakai, J.-I., \& Saito, S. 2005b, New J. Phys., 7, 79 